\title{
STABILITY STUDY OF ETHYLCELLULOSE COATED-TOCOTRIENOL MICROCAPSULES PREPARED BY SOLVENT EVAPORATION AND SPRAY DRYING TECHNIQUES
}

\author{
SILVIA SURINI*, NADIA HUSNUL KHOTIMA \\ Laboratory of Pharmaceutics and Pharmaceutical Technology Development, Faculty of Pharmacy, Universitas Indonesia, Depok, \\ West Java, Indonesia. Email: silvia@farmasi.ui.ac.id
}

Received: 26 September 2019, Revised and Accepted: 17 December 2019

\section{ABSTRACT}

Objective: Tocotrienol is a natural Vitamin E compound with greater antioxidant activity than tocopherol. However, tocotrienol is considered unstable, which limits its handling and use in various product formulations. In this study, to enhance the stability of tocotrienol, tocotrienol oil was converted into a powder through a microencapsulation method using ethylcellulose (EC) as the coating material.

Methods: Tocotrienol microcapsules were formulated with EC in ratios of 1:2 and 1:3 by solvent evaporation (SE) and spray drying techniques. The obtained microcapsules were then characterized in terms of shape and morphology, particle size, entrapment efficiency, percentage yield, flow properties, water content, swelling, and drug release. In addition, stability studies at both room temperature and elevated temperatures were performed.

Results: Our results demonstrated that the tocotrienol microcapsules were of a white-yellowish powder of irregular shape, with particle sizes between $1 \mu \mathrm{m}$ and $60 \mu \mathrm{m}$ and entrapment efficiency of $21.60 \%$ and $99.75 \%$. After 12 weeks of storage at room temperature, the remaining level of tocotrienol in the microcapsules was $96.46-97.74 \%$. In the accelerated stability study at elevated temperatures, the resulting $\mathrm{k}_{25}$ values ranged from $1.02 \times 10^{-5}$ to $1.32 \times 10^{-5} / \mathrm{h}$. Thus, the predicted shelf-life $\left(t_{90}\right)$ of the microencapsulated tocotrienol was determined to be between 11.01 and 14.27 months.

Conclusion: The microencapsulation of tocotrienol with EC using SE and spray drying techniques produced a solid form of tocotrienol that was considerably more stable than the natural form of tocotrienol.

Keywords: Microencapsulation, Solvent evaporation, Spray drying, Stability test, Tocotrienol.

(c) 2020 The Authors. Published by Innovare Academic Sciences Pvt Ltd. This is an open access article under the CC BY license (http://creativecommons. org/licenses/by/4. 0/) DOI: http://dx.doi.org/10.22159/ijap.2020.v12s1.FF045

\section{INTRODUCTION}

Tocotrienol, a member of the Vitamin E family, is a vitamin component in oil form present in high amounts in rice bran, palm, and annatto seeds [1]. Tocotrienol is known to have greater antioxidant activity than tocopherol. Indeed, in rat liver microsomes, $\alpha$-tocotrienol was shown to provide 40 times greater protection against Fe (II) 1 nicotinamide adenine dinucleotide phosphate-induced lipid peroxidation than $\alpha$-tocopherol. Alpha-tocotrienol has also been shown to be 6.5 times more effective in protecting CYP- 450 enzymes from oxidative damage [2]. However, tocotrienol is considerably more unstable compared with tocopherol. One reason for this is that tocotrienol in its natural oil form has three trans double bonds in its side chain, which makes it easily oxidized. In addition, compared with tocopherol, tocotrienol oil is more sensitive to exposure to high heat [3]. As such, the study and use of tocotrienol itself remain rare. Therefore, to use tocotrienol in product formulations, it is necessary to convert it into a stable nutraceutical product. One method that has been used with other compounds is microencapsulation, which can convert a liquid into a solid form. As a technique, microencapsulation is the process of applying a thin coating to small particles of solids or droplets of liquid and dispersions [4].

In this study, tocotrienol oil was microencapsulated through solvent evaporation (SE) and spray dry (SD) techniques using ethylcellulose (EC) coating. The produced microcapsules were characterized and tested for stability with tocotrienol oil as a comparison. In addition, the microcapsules were characterized in terms of shape and morphology, percentage yield, entrapment efficiency, water content, particle size distribution, swelling, flow properties, and drug release. Stability testing was performed to evaluate the physical and chemical stability at both room temperature and elevated temperatures, and the shelf life was determined. Collectively, our findings indicate that the microencapsulation of tocotrienol should facilitate its handling and stability in product formulations.

\section{METHODS}

\section{Materials}

Tocotrienol was purchased from Davos Life Science (Singapore), EC N-10 was purchased from Aqualon (USA), sodium lauryl sulfate (SLS), Span 80 , Tween 80 , liquid paraffin, acetone, ethanol $96 \%$, dichloromethane, and distilled water were purchased from PT. Brataco (Indonesia).

\section{Preparation of tocotrienol microcapsules}

The tocotrienol microcapsules were prepared through emulsion SE (ESE), SE, and SD techniques into eight formulas, as shown in Table 1.

\section{ESE method}

Solution A was prepared from dissolved EC and tocotrienol (T3) in acetone. Solution B was prepared from Span $80(1.5 \%)$ in liquid paraffin (light). Solution A was added into Solution B with a stirring speed of $1500 \mathrm{rpm}$ for $2 \mathrm{~h}$ at $60^{\circ} \mathrm{C}$. Microcapsules were then filtered, washed with SLS solution (7.5\%) and distilled water, and dried [5].

\section{SE method}

Solution A was prepared by dissolving EC and T3 in a mixture of ethanol and dichloromethane (1:1). Solution B was prepared by dissolving Tween $80(0.1 \%)$ in distilled water. Solution A was poured into Solution B, then stirred at a speed of $2000 \mathrm{rpm}$ for $1 \mathrm{~h}$ at room temperature. The microcapsules were collected by filtering and then dried [6]. 


\section{SD method}

Microcapsules were prepared with two types of solvents

a. EC and T3 were dissolved in acetone at a drug/polymer ratio of 1:2 and $1: 3$

b. EC and T3 were dissolved in a mixture of acetone:ethanol (1:2) at a drug/polymer ratio of $1: 2$ and 1:3 [7]

Each solution was sprayed through a mini spray drier B290 (Buchi, Swiss) under the conditions shown in Table 2.

\section{Characterization of microcapsules}

Shape and morphology

Scanning electron microscopy (SEM) (FEI FE-SEM Inspect F50, USA) was used to characterize the surface topography of the microcapsules coated with a gold layer at magnifications of 500, 1000, 3000, and 5000 times [8].

\section{Percentage yield}

The weight of the microcapsules obtained was compared with the total weight of both the drug and polymer used $[8,9]$.

\section{Entrapment efficiency}

An amount of the microcapsules equivalent to $10 \mathrm{mg}$ of T3 was dissolved in a $100-\mathrm{mL}$ volumetric flask using $96 \%$ ethanol. The absorbance was measured at the $\lambda$ maximum of T3 $[8,9]$.

\section{Water content}

One gram of the microcapsules was measured using a moisture analyzer at $105^{\circ} \mathrm{C}[8]$

\section{Particle size distributions}

The microcapsules were dispersed in distilled water then observed with a particle size analyzer (Beckman Coulter, Germany). The particle size distribution curve was based on the particle volume.

Table 1: Formulation of tocotrienol microcapsules

\begin{tabular}{|c|c|c|c|c|c|c|c|c|}
\hline \multirow[t]{3}{*}{ Ingredients } & \multicolumn{8}{|c|}{ Formula } \\
\hline & \multicolumn{2}{|c|}{ ESE } & \multicolumn{2}{|l|}{ SE } & \multicolumn{4}{|l|}{ SD } \\
\hline & F1 & F2 & F3 & F4 & F5 & F6 & F7 & F8 \\
\hline T3 (gr) & 0.5 & 0.5 & 0.5 & 0.5 & 2.5 & 2.5 & 2.5 & 2.5 \\
\hline EC (gr) & 1 & 1.5 & 1 & 1.5 & 5 & 7.5 & 5 & 7.5 \\
\hline Acetone $(\mathrm{mL})$ & 20 & 30 & - & - & 100 & 150 & - & - \\
\hline $\begin{array}{l}\text { Liquid paraffin } \\
\text { (mL) }\end{array}$ & 60 & 90 & - & - & - & - & - & - \\
\hline Span $80(\mathrm{~mL})$ & 0.9 & 1.35 & - & - & - & - & - & - \\
\hline $\begin{array}{l}\text { Dichloromethane: } \\
\text { ethanol (1:1) (mL) }\end{array}$ & - & - & 20 & 30 & - & - & - & - \\
\hline $\begin{array}{l}\text { Distilled } \\
\text { water }(\mathrm{mL})\end{array}$ & - & - & 100 & 150 & - & - & - & - \\
\hline Tween $80(\mathrm{~mL})$ & - & - & 0.1 & 0.15 & - & - & - & - \\
\hline $\begin{array}{l}\text { Acetone:ethanol } \\
(1: 2)(\mathrm{mL})\end{array}$ & - & - & - & - & - & - & 100 & 150 \\
\hline $\begin{array}{l}\text { Drug-polymer } \\
\text { ratio }\end{array}$ & $1: 2$ & $1: 3$ & $1: 2$ & $1: 3$ & $1: 2$ & $1: 3$ & $1: 2$ & $1: 3$ \\
\hline
\end{tabular}

ESE: Emulsion solvent evaporation, SE: Solvent evaporation, SD: Spray dry

Table 2: Optimum spraying conditions

\begin{tabular}{lllll}
\hline Solution & \multicolumn{2}{l}{ Temperature $\left({ }^{\circ} \mathbf{C}\right)$} & $\begin{array}{l}\text { Feed flow } \\
\text { (ml/min) }\end{array}$ & $\begin{array}{l}\text { Pressure } \\
\text { (bar) }\end{array}$ \\
\cline { 2 - 5 } & Inlet & Outlet & & \\
\hline Acetone & 80 & 70 & 5 & 3 \\
Acetone:ethanol (1:2) & 90 & 80 & 5 & 3 \\
\hline
\end{tabular}

Microcapsule flow properties

The compressibility index of the microcapsules was tested with a bulk-tapped density tester. The microcapsule volume after tapping 300 times was measured. The flow rate was calculated using a flow meter. The repose angle was measured from the steepest angle of the microcapsules.

\section{Swelling test}

The microcapsules $(100 \mathrm{mg}$ ) were added to $10 \mathrm{~mL}$ of medium (distilled water and phosphate buffer $\mathrm{pH}$ 6.8) in a centrifuge tube and immersed for 60 and $120 \mathrm{~min}$. After centrifugation (4000 rpm), the supernatant was removed, and the final weight was determined [10]

\section{Drug release study}

The microcapsules release profile was evaluated in $100 \mathrm{~mL}$ phosphate buffer medium pH 6.8 added to $100 \mathrm{mg}$ SLS $(0.1 \%)$ to help the T3 dissolve. T3 release was carried out at $37 \pm 0.5^{\circ} \mathrm{C}$ with an agitation speed of $100 \mathrm{rpm}$ for $8 \mathrm{~h}$. Five milliliters aliquots of the samples were taken at $5,15,30,45,60,90,120,180,240,300,360,420$, and $480 \mathrm{~min}$. The percentage of released $\mathrm{T} 3$ was calculated. T3 levels were determined by spectrophotometry at the $\lambda$ maximum [10].

\section{Stability study}

Room temperature stability study

The stability of the microcapsules F1-F8 was compared with T3 oil at room temperature $\left( \pm 25^{\circ} \mathrm{C}\right)$ for 12 weeks. Organoleptic observation and T3 level determination using a spectrophotometer were performed on each sample every week.

\section{Accelerated stability study}

The microcapsules F1-F8 were compared with T3 oil to observe stability and shelf life. The samples were placed in vial bottles and tested at 80,90 , and $100^{\circ} \mathrm{C}$. At each temperature, sample aliquots were taken at 1, 2, 3, 4, 6, and $8 \mathrm{~h}$. T3 levels in each sample were measured by spectrophotometry.

\section{RESULTS AND DISCUSSION}

Characterization of microcapsules Shape and morphology

Through microencapsulation, T3 was successfully converted from an oil into a powder form with EC by the ESE, SE, and SD methods. The SEM examination was performed on four types of microcapsules to be representative of each method and to observe its surface morphology.
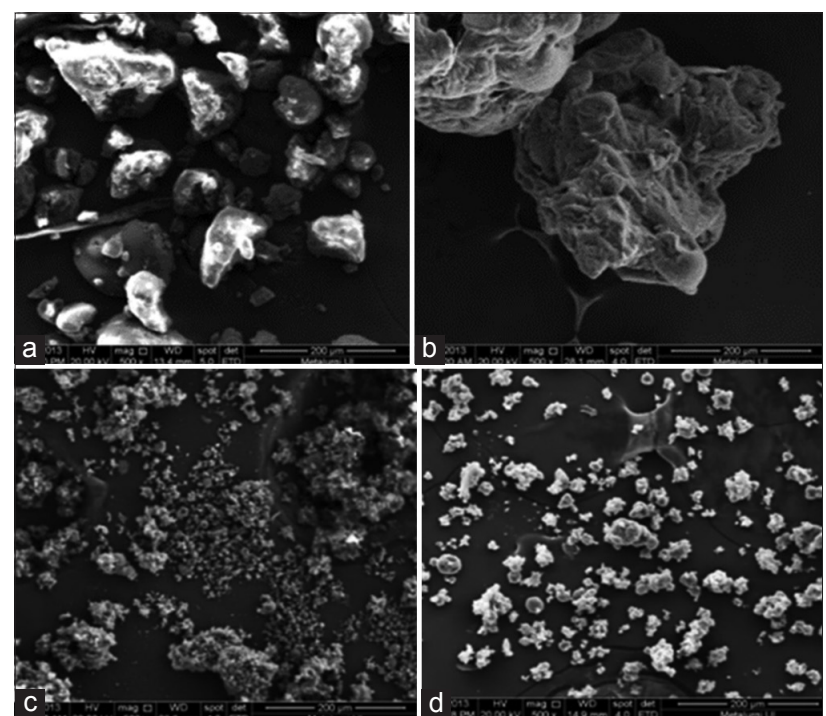

Fig. 1: Microphotographs of the tocotrienol microcapsules (a) F2, (b) F4, (c) F6, and (d) F8 at a 500x 
Test results are shown in Fig. 1. The shape of the microcapsules was not spherical but rather varied due to the different methods of manufacture. The shape produced by the ESE and SE methods appeared to depend on the droplet and stirring speeds. The F8 formulation, which was produced through the SD method with acetone: ethanol (1:2), was not spherical; however, the F6 formulation prepared with acetone as the solvent was round and spherical. The surface morphology of all the microcapsules appeared porous; this factor can affect T3 release.

\section{Percentage yield}

The test results are shown in Table 3 . The percentage yield value showed good results, ranging from 62.46 to $87.23 \%$. The percentage yield value of the microcapsules produced by the ESE method was lower because a lot of T3 was not trapped. The percentage yield value of the microcapsules produced by the SD method was generally quite good considering a lot of the material was lost during the drying process. This can be explained by the EC coating ability and optimal inlet/outlet temperature settings.

\section{Entrapment efficiency}

The results are shown in Table 3, with entrapment efficiency ranging from 21.60 to $99.75 \%$. The lowest value was obtained from microcapsules in the F1 and F2 formulations, at $21.60 \%$ and $27.48 \%$, respectively, while the six other formulas demonstrated high values in the range of $91.06 \%-99.75 \%$. The low entrapment efficiency values of F1 and F2 were likely caused by the ESE method, which is considered less suitable for oil materials. The T3 oil has properties as such that it can be mixed with other oils, such as paraffin oil. It has also been described that drug loss into the continuous phase occurs while the dispersed phase stays in a transitional, semi-solid state. If a drug is more soluble in the continuous phase than in the dispersed phase, it will easily diffuse into the continuous phase during this stage [8]. Entrapment efficiency values were higher for microcapsules with a higher coating concentration. The entrapment efficiency of other microcapsules showed high values, indicating the suitability of the SE and SD methods.

Table 3: Percentage yield, entrapment efficiency, water content, and particle size value of microcapsules F1-F8

\begin{tabular}{|c|c|c|c|c|c|}
\hline \multirow[t]{2}{*}{ Microcapsule formulation } & \multirow[t]{2}{*}{ Percentage yield (\%) } & \multirow[t]{2}{*}{ Entrapment efficiency (\%) } & \multirow[t]{2}{*}{ Water content (\%) } & \multicolumn{2}{|c|}{ Particle size $(\mu \mathrm{m})$} \\
\hline & & & & Mean & Median \\
\hline F1 & 65.36 & $21.60 \pm 1.95$ & $4.71 \pm 0.42$ & 15.49 & 14.44 \\
\hline F3 & 82.38 & $99.30 \pm 0.62$ & $4.63 \pm 0.33$ & 19.96 & 21.15 \\
\hline $\mathrm{F} 4$ & 87.23 & $99.67 \pm 1.69$ & $4.46 \pm 0.53$ & 20.80 & 23.11 \\
\hline F5 & 74.74 & $91.06 \pm 0.73$ & $3.65 \pm 0.07$ & 22.04 & 24.54 \\
\hline F6 & 86.35 & $99.25 \pm 0.14$ & $3.97 \pm 0.06$ & 24.17 & 25.99 \\
\hline F7 & 62.46 & $99.15 \pm 0.82$ & $4.06 \pm 0.04$ & 21.93 & 25.02 \\
\hline F8 & 75.20 & $99.75 \pm 0.12$ & $5.55 \pm 0.05$ & 29.59 & 30.93 \\
\hline
\end{tabular}
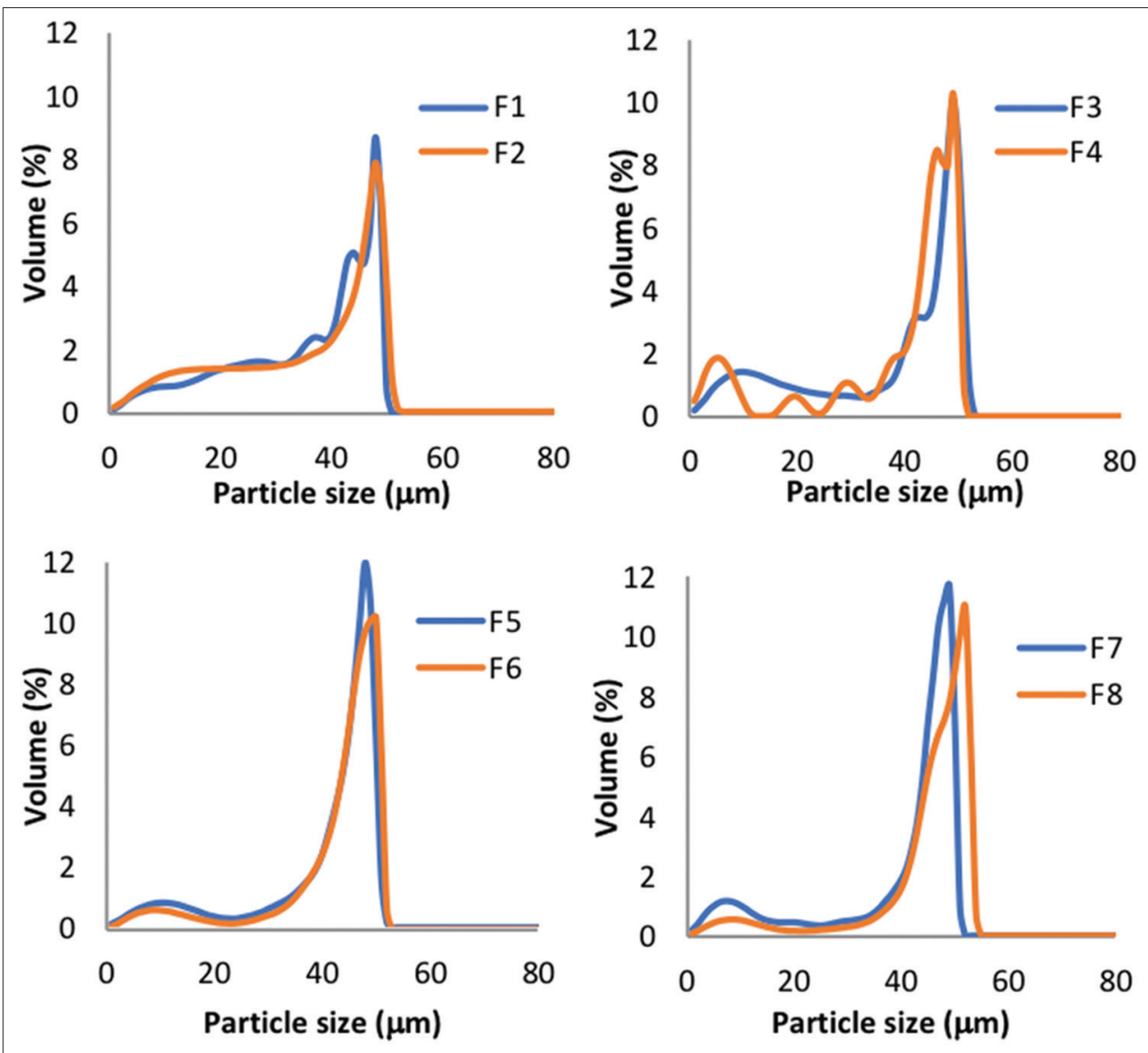

Fig. 2: Particle size distribution of microcapsules F1-F8 
Particle size distribution

The microcapsules particle size distribution chart in Fig. 2 demonstrates that the microcapsules sizes ranged from $1 \mu \mathrm{m}$ to $60 \mu \mathrm{m}$. Test results listed in Table 3 showed that the particle size of the microcapsules F1-F8 had value ranges for the mean and median from $14.44 \mu \mathrm{m}$ to $30.93 \mu \mathrm{m}$ and $15.49 \mu \mathrm{m}$ to $29.59 \mu \mathrm{m}$, respectively. McNamee et al. [11] reported that microcapsule sizes produced by the SD method are affected by solution viscosity, feed flow, and nozzle size [12]. The size of the microcapsules produced by the SE and ESE methods decreased with increasing stirring time. Indeed, it has been reported that the particles get smaller, while the particle distribution becomes narrower, with increasing stirring rates and times because the particles become separated in a stable form without aggregation [13].

\section{Water content}

The test results are shown in Table 3. Based on the test, all formulas of the microcapsules had a relatively low water content, which ranged between $3.65 \%$ and $6.36 \%$. The water in the microcapsules is believed to be derived from the distilled water during their preparation. EC is stable and not affected by water so the microcapsules are not considered hygroscopic.

\section{Microcapsules flow properties}

In general, all microcapsules indicated poor flow properties. Among the F1-F8 formulations, the best flow properties were demonstrated by F5 and F7. Flow properties can be determined through the angle of repose, the compressibility index, and the Hausner ratio. Microcapsules in the F1 and F2 formulations could not flow. The F5 and F7 formulations showed very good flow properties with an angle of repose $<30^{\circ}$, while the F3, F4, F6, and F8 formulations were considered good with an angle of repose between 31 and $35^{\circ}$. However, based on the compressibility index and Hausner ratio values, only the F7 and F5 formulations were considered good, while the other formulas did not meet the requirements of good flow properties.

\section{Swelling test}

The microcapsule swelling index ranged from $76.27 \%$ to $318.06 \%$ in distilled water, while that in phosphate-buffered ranged from $126.02 \%$ to $340.62 \%$. The low swelling index values can be explained by the EC coating with hydrophobic properties. As such, the microcapsules with a higher coating concentration showed higher swelling index values. Particle size also affects the swelling index, with the F1 and F2 formulas having a smaller size compared with the other formulas.

\section{Release study}

The dissolution profiles of the microcapsules in the F1-F8 formulations are shown in Fig. 3. T3 was released from all microcapsules produced by the SD method, with the fastest release by F7 at $3 \mathrm{~h}$, followed by F5 at 3.5 h, F8 at 5 h, and F6 at 6.5 h. F3 and F4 produced by the SE method could only release all the T3 after 5 and $8 \mathrm{~h}$, respectively. This is in contrast to the F1 and F2 formulations, which only was able to release T3 at $67.61 \%$ and $54.46 \%$, respectively, after testing for $8 \mathrm{~h}$. After $3.5 \mathrm{~h}, \mathrm{~F} 7$ and F5 released all the T3, while F6 and F8 only released T3 as much as 79.93\% and $60.09 \%$, respectively. Slower release from F6 to F8 was likely due to the larger particle sizes. In general, microcapsules produced by the SD method showed a faster release than other methods due to the small and uniform particle size. F3 and F4 produced by the SE method could only release all the T3 after 5 and $8 \mathrm{~h}$, respectively. The release of F3 was faster due to the decreased coating concentration compared with F4. F1 and F2 produced by the ESE method showed the slowest dissolution rate among the formulas. For F1 and F2, the decreased release of T3 was likely influenced by the low number of T3 molecules trapped in the microcapsules. In addition, despite the particle size being not much different from the other formulas, the reduced T3 content in F1 and F2 resulted in a greater coating concentration; thus diffusion was slower. Furthermore, microcapsules with different coating concentrations are known to have different wall thicknesses, with those of smaller

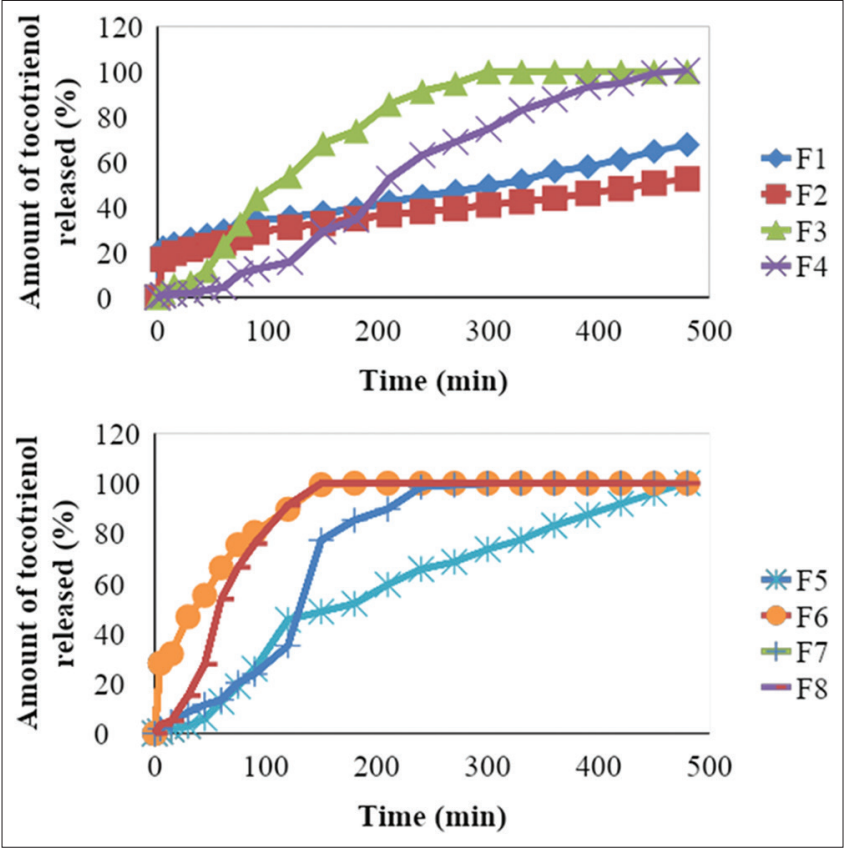

Fig. 3: Drug release profiles of the tocotrienol microcapsules F1-F8 in phosphate buffer (pH 6.8)

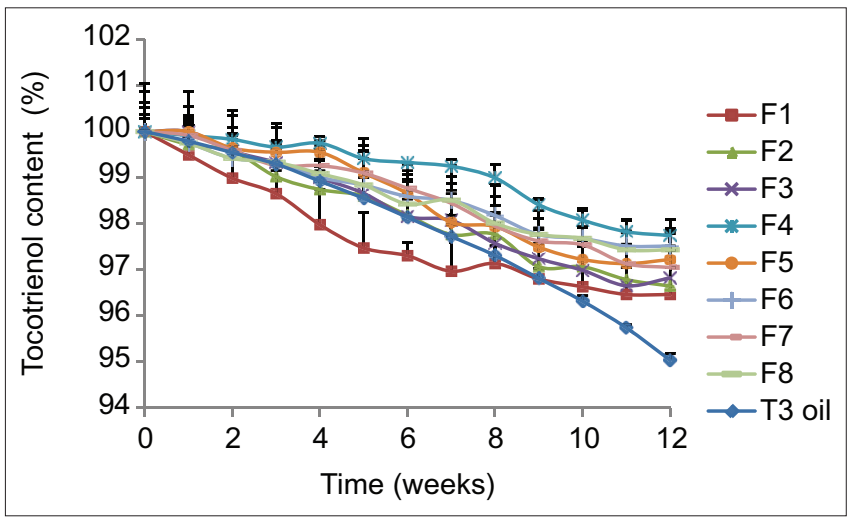

Fig. 4: Stability study of the tocotrienol microcapsule F1-F8 formulations and T3 oil at room temperature for 12 weeks

particle size having thinner walls. For EC as a water-insoluble polymer, drug release is mainly driven by permeation through the hydrophobic membrane within water-filled pores [14].

\section{Stability study}

Stability study at room temperature

Changes in T3 levels in the microcapsule F1-F8 formulations and T3 oil are shown in Fig. 4. T3 levels in the microcapsules and those of the non-encapsulated T3 after 12 weeks storage at room temperature decreased, with remaining T3 levels at $95.04 \%$ for the oil form and $97.74 \%$ to $96.46 \%$ for the F1-F8 microcapsule formulations. Stability from the lowest to the highest $\mathrm{T} 3$ remaining concentration was: $\mathrm{T} 3$ oil $>$ F $1>$ F2 $>$ F $3>$ F $7>$ F5 $>$ F $8>$ F6 $>$ F4

F1 and F2 formulations produced by the ESE method showed the lowest stability among the formulas. F3-T3 levels also decreased significantly compared with the F4 formulations due to a decreased coating concentration. F4-F8 formulations produced by the SD method showed good chemical stability.

The content of T3 oil at room temperature decreased by $4.96 \%$ more than the microcapsule F1-F8 formulations. This value indicated that T3 
Table 4: $k_{25}, t_{90}, t_{1 / 2}$, and Ea values of microcapsules F1-F8 and T3 oil

\begin{tabular}{lllll}
\hline Formula & $\mathbf{k}_{\mathbf{2 5}} \times \mathbf{1 0}^{-\mathbf{5}}\left(\mathbf{h}^{-\mathbf{1}}\right)$ & $\mathbf{t}_{\mathbf{9 0}}$ (months) & $\mathbf{t}_{\mathbf{1} / \mathbf{2}}$ (years) & Ea (kcal/mol) \\
\hline F1 & $1.31 \pm 0.03$ & $11.14 \pm 0.24$ & $6.30 \pm 0.13$ & $18.42 \pm 0.27$ \\
F2 & $1.18 \pm 0.06$ & $12.41 \pm 0.65$ & $6.83 \pm 0.36$ & $18.75 \pm 0.28$ \\
F3 & $1.32 \pm 0.03$ & $11.01 \pm 0.28$ & $6.06 \pm 0.16$ & $21.00 \pm 0.14$ \\
F4 & $1.08 \pm 0.06$ & $13.49 \pm 0.77$ & $7.42 \pm 0.42$ & $20.07 \pm 0.17$ \\
F5 & $1.09 \pm 0.04$ & $13.38 \pm 0.52$ & $7.36 \pm 0.29$ & $20.08 \pm 0.19$ \\
F6 & $1.04 \pm 0.05$ & $14.06 \pm 0.76$ & $7.73 \pm 0.42$ & $19.19 \pm 0.26$ \\
F7 & $1.15 \pm 0.02$ & $12.73 \pm 0.24$ & $7.00 \pm 0.13$ & $19.86 \pm 0.02$ \\
F8 & $1.02 \pm 0.01$ & $14.27 \pm 0.20$ & $7.85 \pm 0.11$ & $19.53 \pm 0.01$ \\
T3 oil & $2.02 \pm 0.28$ & $7.30 \pm 0.96$ & $4.02 \pm 0.53$ & $19.27 \pm 0.39$ \\
\hline
\end{tabular}

in oil form degraded faster than its solid form, which is consistent with the presence of the double bonds which are easily oxidized.

Stability in solid form can be affected by humidity, temperature, excipients, oxygen, and light. Moisture can also affect stability if the active substance is water-soluble and a hygroscopic excipient is used. Due to the humidity, a hydrolysis reaction can occur and increase the degradation rate of the active substance [15].

\section{Accelerated stability study}

The study was conducted at three different temperatures to determine the T3 degradation rate of the microcapsules F1-F8. The temperature selection was optimized at 80,90 , and $100^{\circ} \mathrm{C}$, which can result in decreased levels of $\mathrm{T} 3$ so that the rate constant can be calculated.

The results are shown in Table 4 and shown that the $\mathrm{k}_{25}, \mathrm{t}_{90}, \mathrm{t}_{1 / 2}$, and Ea values varied. The $\mathrm{k}_{25}$ values of the F1-F8 microcapsules were ranged from $1.02 \times 10^{-5}$ to $1.32 \times 10^{-5} / \mathrm{h}$, while that of the T3 oil was $2.02 \times 10^{-5} / \mathrm{h}$. The degradation rate at $25^{\circ} \mathrm{C}$ from the lowest to the highest was: F8 $>\mathrm{F} 6$ $>$ F4 $>$ F5 $>$ F7 $>$ F2 $>$ F1 $>$ F3 $>$ T3 oil.

Common degradation reactions include hydrolysis and oxidation reactions. In a solid form such as microcapsules, oxidation can occur from hydrolysis in the solid-state only when the active substance is water-soluble. Oxidative degradation can occur spontaneously under oxygen influence without a catalyst. This reaction occurs in unsaturated oils such as T3 [16]. As researched by Simonne and Eitenmiller [17], T3 degrades rapidly in oil form due to the saturated fatty acid content. This happens when the polyunsaturated fatty acids content in an oil is low such that the T3 becomes the main substrate that reacts with the oxygen [3]. The shelf life and half-life values of the microcapsule F1-F8 formulations and the T3 oil followed the reverse order of the $\mathrm{k}_{25}$ value, with the lowest to highest being $\mathrm{T} 3$ oil $>\mathrm{F} 3>\mathrm{F} 1>\mathrm{F} 2>\mathrm{F} 7>\mathrm{F} 5>\mathrm{F} 4>\mathrm{F} 6$ $>$ F8. The F1-F8 shelf life value ranged from 11.01 to 14.27 months with a half-life value of 6.06 to 7.85 years, while those of the T3 oil were 7.30 months and 4.02 years, respectively.

\section{CONCLUSION}

Based on this study, it can be concluded that T3 oil can be converted to a solid form using EC coating in conjunction with SD and SE methods. The characteristic microcapsules were a yellowish powder, with a particle size of 1-60 $\mu \mathrm{m}$ and entrapment efficiency of $21.60 \%-99.75 \%$; the best results were obtained from microcapsules produced by the SD method. In addition, it can be concluded that the T3 microcapsules showed better stability than the T3 oil, particularly for the F6 and F8 formulations. After 12 weeks of storage at room temperature, T3 levels in the microcapsules ranged from $96.46 \%$ to $97.74 \%$. The shelf life value of the microcapsule F1-F8 formulations was 11.01-14.27 months.

\section{ACKNOWLEDGMENTS}

The authors gratefully acknowledge Universitas Indonesia for support and a PITTA research grant 2019.

\section{CONFLICTS OF INTEREST}

The authors declare that they have no conflicts of interest.

\section{REFERENCES}

1. Aggarwal BB, Sundaram C, Prasad S, Kannappan R. Tocotrienols, the Vitamin $\mathrm{E}$ of the $21^{\text {st }}$ century: Its potential against cancer and other chronic diseases. Biochem Pharmacol 2010;80:1613-31.

2. Packer L, Weber SU, Rimbach G. Molecular aspects of alpha-tocotrienol antioxidant action and cell signalling. J Nutr 2001;131:369S-73S.

3. Rossi M, Alamprese C, Ratti S. Tocopherols and tocotrienols as free radical-scavengers in refined vegetable oils and their stability during deep-fat frying. Food Chem 2006;102:812-7.

4. Bakan JA, Anderson J. Microencapsulation. In: Lachman L, Liebermann H, Kanig J, editors. The Theory and Practice of Industrial Pharmacy. Vol. 3. Philadelphia, PA: Lea and Febiger; 1970. p. 384-407.

5. Pachuau L, Mazumder B. A study on the effects of different surfactants on ethylcellulose microspheres. Int J Pharmtech Res 2009;1:966-71.

6. Pande AV, Vaidya PD, Arora A, Dhoka MV. In vitro and in vivo evaluation of ethyl cellulose based floating microspheres of cefpodoxime proxetil. Int J Pharm Biomed Res 2010;1:122-8.

7. Emami J, Varshosaz J. Ahmadi F. Preparation and evaluation of a liquid sustained-release drug delivery system for theophylline using spraydrying technique. Res Pharm Sci 2007;2:1-11.

8. Eduard A. Influence of key parameters on the morphology of ethylcellulose microcapsules prepared via room-temperature spray drying. Cellulose 2010;17:1-10.

9. Panda S, Pattnaik S, Maharana L, Botta GB, Mohapatra P. Formulation and evaluation of zidovudine loaded olibanum resin microcapsules: Exploring the use of natural resins as a biodegradable polymeric material for controlled release. Asian J Pharm Clin Res 2013;6:191-6.

10. Surini S, Anggriani V, Anwar E. Study of mucoadhesive microspheres based on pregelatinized cassava starch succinate as a new carrier for drug delivery. J Med Sci 2009;9:249-56.

11. Jyothi NV, Prasanna PM, Sakarkar SN, Prabha KS, Ramaiah PS, Srawan GY. Microencapsulation techniques, factors influencing encapsulation efficiency. J Microencapsul 2010;27:187-97.

12. McNamee BF, O'Riordan ED, O'Sullivan M. Emulsification and microencapsulation properties of gum Arabic. J Agric Food Chem 1998;46:4551-5.

13. Xie Y, Zhou H, Liang X, He B, Han X. Study on the morphology, particle size and thermal microencapsulated by starch octenyl succinate. Agric Sci China 2010;9:1058-64.

14. Fan-Long J, Soo-Jin P. Preparation and characterization of biodegradable antibiotic-containing poly (E-caprolactone) microcapsules. J Ind Eng Chem 2007;13:608-13.

15. Murtaza G, Ahmad M, Khan SA. Release behavior of different physicochemical properties drug models from the ethylcellulose microcapsules. J Pharm Bioallied Sci 2010;2:153.

16. Florence AT, Attwood D. Physicochemical Principles of Pharmacy. USA: Pharmaceutical Press; 2006. p. 4

17. Simonne AH, Simonne EH, Eitenmiller RR. Retention of Vitamin E and added retinyl palmitate in selected vegetable oils during deep-fat frying and in fried breaded products. J Agric Food Chem 1998;46:5273-7. 